\title{
Solvent Effect on Fluorescence Quenching of 7, 8 Benzo-4-azido Methyl Coumarin by Aniline
}

\author{
R M Melavanki,* N R Patil,† D Nagaraja,ł H D Patil,§ \\ J S Kadadevarmath** and R A Kusanur ††
}

\begin{abstract}
Fluorescence quenching of biologically active studies of 7 , 8 benzo-4-azidomethyl coumarin (7BAMC) by aniline in four different organic solvents namely benzene, dioxane, tetrahydrofuran and acetonitrile has been carried out at room temperature with a view to understand the quenching mechanisms. The Stern-Volmer (S-V) plot has been found to be non-linear with a positive deviation for all the solvents studied. In order to interpret these results we have invoked the ground state complex formation and sphere of action static quenching models. Using these models various quenching rate parameters have been determined. The magnitudes of these parameters suggest
\end{abstract}

\footnotetext{
* Department of Physics, M S Ramaiah Institute of Technology Bangalore560054, Karnataka, India; melavanki73@gmail.com

† Department of Physics, B V B College of Engineering and Technology, Hubli-580031, Karnataka, India

‡ Department of Physics, Bangalore Institute of Technology, Bangalore560004, Karnataka, India

$\S$ Department of Physics, Basaveshwar Science College, Bagalkot-587101, Karnataka, India
}

* Department of Physics, Karnatak University Dharwad-580003, Karnataka, India

tt Department of Chemistry, R V College of Engineering, Bangalore560059, Karnataka, India 


\begin{abstract}
that sphere of action static quenching model agrees well with the experimental results. Hence the positive deviation is attributed to the static and dynamic quenching. Further, with the use of Finite Sink approximation model, it was possible to check these bimolecular reactions as diffusion-limited and to estimate independently distance parameter $R^{\prime}$ and mutual diffusion coefficient $D$. Finally an effort has been made to correlate the values of $R^{\prime}$ and $D$ with the values of the encounter distance $R$ and the mutual coefficient $D$ determined using the Edward's empirical relation and Stokes - Einstein relation.
\end{abstract}

Keywords: Fluorescence quenching, 7,8 benzo-4-azidomethyl coum arin, diffusion limited, static and dynamic quenching and finite sink approximation model.

\title{
1. Introduction
}

Fluorescence quenching of organic molecules in solution by various quenchers like carbon tetrachloride, bromobenzene, aniline, halide ions, metal ions etc. has been studied by several investigators [110]. The study has been mainly to understand the nature of bimolecular reactions taking place both under steady state and transient conditions. This study has not only been of importance in physical sciences but also in chemical, biological and medical sciences [11-16]. The fluorescence yield in bimolecular liquid systems is hindered due to several mechanisms such as static and dynamic quenching, excimer and exciplex formation, charge transfer processes, etc. One of the well known experimental techniques used to study the role of fluorescence quenching is to determine the quenching rate parameter using Stern-Volmer (S-V) plots. If the quenching mechanism is mainly due to dynamic process, then it will be largely due to diffusion in which case diffusion rate parameter $\mathrm{k}_{\mathrm{d}}$ equals the quenching rate parameter $\mathrm{k}_{\mathrm{q}}$ $\left(=\mathrm{K}_{\mathrm{sv}} / \tau\right)$ where $\mathrm{K}_{\mathrm{sv}}$ is the slope of the linear S-V plot and $\tau$ is the decay time of the solute in the absence of the quencher. On the other hand, if the experimental results do not simulate with the simple linear Stern-Volmer relationship, then it may be due to one of the above processes other than or along with diffusion process. 
Coumarins establish a family of dyes $[5-7,10]$ that are applicable in different fields of science and technology. They exhibit strong fluorescence in the UV and VIS region that makes them suitable to use as a colorants, dye laser media and as nonlinear optical chromophores. In medicine, coumarin derivatives are used as anticoagulants, as a fluorescent indicators for the physiological $\mathrm{pH}$ region and as fluorescent probes to determine the rigidity and fluidity of living cells and its surrounding medium. Coumarins and its derivatives have been a subject of considerable interest in numerous fields. Due to the interesting properties associated with coumarins, we undertook the fluorescence studies of newly synthesized 7,8 benzo-4-azidomethyl coumarin. In the present study we have used steady state experimental setup to investigate quenching of 7BAMC by aniline in four different solvents with a view to understand the nature of quenching mechanism involved in these systems.

\section{Theory}

The dynamic process in which quenching mechanism is mainly due to collision is governed by the linear Stern-Volmer (S-V) equation.

$$
\frac{I_{0}}{I}=1+K_{S V}[Q]
$$

Where $I_{0}$ is fluorescence intensity of solute in the absence of quencher and $\mathrm{I}$ is the fluorescence intensity in the presence of quencher, $\mathrm{KSV}$ ( $=\mathrm{kq} \tau$ where $\mathrm{kq}$ is quenching rate parameter) is the S-V constant and $\tau$ is the lifetime of the solute molecule in the absence of quencher. Eq. (1) is applicable as long as the experimental results show linear variation. The departure from linearity in $\mathrm{S}-\mathrm{V}$ plot suggests that quenching mechanism is not purely collisional and this may be due to sphere of action static quenching model [1-2].

According to "Sphere of action static quenching model" the instantaneous or static quenching occurs if the quencher molecule is very near to, or in contact with the fluorescent molecule at the exact moment it happens to be excited. This was explained by the fact that only a certain fraction ' $W$ ' of the excited state is actually 
quenched by the collisional mechanism. Some molecules in the excited state, the fraction of which is $(1-\mathrm{W})$, are de-activated almost instantaneously after being formed because a quencher molecule happens to be randomly positioned in the proximity at the time the molecules are excited and interacts very strongly with them. Several models were employed (Somoluchowski model) to describe this static quenching process, all leading to the following modified form of the S-V equation [1-2].

$$
\frac{I_{0}}{I}=\frac{1+K_{S V}[Q]}{W}
$$

where $\mathrm{K}_{\mathrm{sv}}$ has its usual meaning as explained earlier and [Q] is the quencher concentration. The Somluchoski's diffusion controlled equation containing transient term is written as.

$$
K_{d}=4 \pi N^{\prime} D R+4 R^{2} N^{\prime}(\pi D)^{1 / 2} t^{-1 / 2}
$$

where $\mathrm{N}^{\prime}$ is the Avogadro's number per millimole, $\mathrm{R}$ is the encounter distance, i.e. the sum of the radii of the diffusion coefficients of solute and quencher molecules and $t$ is the time. The retention of the later term of equation (3) leads to an additional factor, $W$ in equation (2). This additional factor $W$ is given by [1-2]

$$
\begin{gathered}
W=\exp (-V[Q]) \\
\operatorname{In}(1 / W)=V[Q]
\end{gathered}
$$

where $\mathrm{V}$ is the static quenching constant and it represents an active volume element surrounding the excited solute molecule.

Instantaneous (static) quenching occurs in a randomly distributed system when a quencher happens to reside within a sphere of action with a volume ' $\mathrm{V} / \mathrm{N}^{\prime}$, and radius ' $\mathrm{r}$ ' i.e.

$$
V / N^{\prime}=\left(4 \pi r^{3}\right) / 3
$$

surrounding a solute molecule at the time of excitation.

As $\mathrm{W}$ depends on the quencher concentration $[Q]$ the $S-V$ plots for a quencher with a high quenching ability generally deviate from linearity. Thus it is worth rewriting equation (2) as 


$$
\frac{\left[1-\left(I / I_{O}\right)\right]}{[Q]}=K_{S V}\left[\frac{I}{I_{O}}\right]+\frac{(1-W)}{[Q]}
$$

From equation (6) one can easily calculate $\mathrm{K}_{\mathrm{sv}}$ and the values of $\mathrm{W}$ by least square fit method. The static quenching constant $\mathrm{V}$ and the values of ' $r$ ' of sphere of action can also be calculated using the equations (4), (5) and (6). According to Andre et al. [11] and Zeng et al. [16], if the distance between the quencher molecules and the excited molecule lies between the encounter distance and the kinetic distance, the static effect takes place especially in the case of steady state experiments irrespective of the ground state complex formation provided the reactions are limited by diffusion. In order to find whether the reactions are diffusion limited one can invoke the finite sink approximation model.

\section{Finite Sink Approximation model}

Keizer [17-19] has proposed a non equilibrium statistical modification of the Smoluckhowski-Collins-Kimball (SCK) expression to fit the $\mathrm{I}_{0} / \mathrm{I}$ ratio in fluorescence quenching. In the case of SCK model time-dependent rate coefficient $\mathrm{k}(\mathrm{t})$ for diffusion limited reaction of initially randomly distributed reactants is given by [20]

$$
k(t)=a+b \exp \left(c^{2} t\right) \operatorname{erfc}\left(c t^{1 / 2}\right)
$$

Where

$$
\begin{aligned}
& a=k_{a}\left[1+\frac{k_{a}}{4 \pi N^{\prime} R D}\right]^{-1} \\
& b=k_{a}\left[1+\frac{4 \pi N^{\prime} R D}{k_{a}}\right]^{-1} \\
& c=\left[1+\frac{k_{a}}{4 \pi N^{\prime} R D}\right] \frac{D^{1 / 2}}{R}
\end{aligned}
$$


Integration of equation (7) between the limits (Q) $(\infty)$ at $r \rightarrow \infty$ and [Q] (R) at $r=R$ provides the well known expression

$$
1 / k_{q}=\left(1 / k_{d}\right)+\left(1 / k_{a}\right)
$$

where $k_{d}=4 \pi N^{\prime} R D$ and $k_{a}$ is the activation energy controlled rate constant describing the reaction of encountered pairs at a reactive distance R. D is the sum of the diffusion coefficients of solute and quencher molecules. Following eqn. (11), $\mathrm{k}_{\mathrm{q}}$ is independent of [Q]. But for efficient quenching process in liquids, $\mathrm{k}_{\mathrm{q}}$ is often observed to increase with [Q]. This might be attributed to static quenching of solute molecule in the vicinity of [Q] and transient effects arising from an initial time dependence of the concentration gradient or combination of them [16].

But if one assumes that only the first encounter is of interest in the case of efficient fluorescence quenching an initial average separation distance $r_{0}$ can be defined (sink radius) so that the diffusive region of interest for a first encounter is in the range $R$ $\leq r \leq r_{o}$ such that all subsequent encounters are eliminated. Integration of the flux equation between the limits $[Q]\left(r_{o}\right)$ at $r_{o}$ and $[Q](R)$ at $\mathrm{R}$ leads to modification of expression (11) as

$$
\frac{1}{k_{q}}=\frac{1-\left(R / r_{o}\right)}{k_{d}}+\frac{1}{k_{a}}
$$

This equation reduces to the reaction limited form $\left(k_{q}=k_{a}\right)$ both for inefficient quenching $\left(\mathrm{k}_{\mathrm{a}}<<\mathrm{k}_{\mathrm{d}}\right)$ and for quenching in pure quenching solvents where $R=r_{0}$. In the diffusion controlled limit $\left(k_{a}>>k_{d}\right)$ equation (12) reduces to

$$
k_{q}=\frac{k_{d}}{1-R / r_{o}}
$$

and $\mathrm{k}_{\mathrm{q}}$ depends on the quenching concentration through $\mathrm{r}_{\mathrm{o}}$. Since the sink radius $\left(\mathrm{r}_{\mathrm{o}}\right)$ is identified with the most probable nearest neighbour initial separation, the appropriate distribution requires that [18] $\mathrm{r}_{\mathrm{o}}=\left(2 \pi \mathrm{N}^{\prime}[\mathrm{Q}]^{-1 / 3}\right)$ Replacing $\mathrm{r}_{\mathrm{o}}$ in eqn. (12) by $\left(2 \pi \mathrm{N}^{\prime}[\mathrm{Q}]^{-1 / 3}\right)$ and $\mathrm{k}_{\mathrm{d}}$ by its value (i.e. $4 \pi \mathrm{N}^{\prime} \mathrm{RD}$ ) and dividing everywhere by the 
fluorescence lifetime of solute in the absence of quencher $\left(\tau_{0}\right)$ one obtains the modified S-V relationship as [5-7],

$$
K_{S V}^{-1}=\left(K_{S V}^{O}\right)^{-1}-\frac{\left.2 \pi N^{\prime}\right)^{1 / 3}}{4 \pi N^{\prime} D \tau}[Q]^{1 / 3}
$$

where $\mathrm{KO}_{\mathrm{SV}}=4 \pi \mathrm{N}^{\prime} \mathrm{RD} \tau \mathrm{k}_{\mathrm{a}} /\left(4 \pi \mathrm{N}^{\prime} \mathrm{RD}+\mathrm{k}_{\mathrm{a}}\right) \mathrm{A}$ plot of $\mathrm{K}^{-1} \mathrm{SV}$ against $[\mathrm{Q}]^{1 / 3}$ becomes linear with negative slope. Mutual diffusion coefficient $D$ becomes directly accessible from the slope of the graph exemplified in equation (14) and $\mathrm{K}_{\mathrm{SV}}$ is obtained at $[\mathrm{Q}]=0$ regardless of the relative magnitudes of $\mathrm{k}_{\mathrm{a}}$ and $\mathrm{k}_{\mathrm{d}}\left(=4 \pi \mathrm{N}^{\prime} \mathrm{RD}\right)$, whether quenching is diffusion limited or not. $\mathrm{KO}_{\mathrm{Sv}}$ can also be written as

$$
K_{S V}^{O}=4 \pi N^{\prime} D R^{\prime} \tau
$$

where $R^{\prime}$ is the distance parameter and has the same meaning as in the long time SCK model and is given as

$$
R^{\prime}=R\left[1+4 \pi N^{\prime} D R / k_{a}\right]^{-1}
$$

Then according to the theory discussed above if ka is greater than $k_{d}$ [i.e. eqn. (11)] then the reactions are said to be diffusion limited [16] (i.e. for $\mathrm{R}^{\prime}<\mathrm{R}$ ). But for $\mathrm{R}^{\prime}>\mathrm{R}$, the bimolecular reactions of fluorescence quenching are said to be diffusion limited, if the values of $\mathrm{k}_{\mathrm{q}}$ determined from equation (6) are greater than $4 \pi$ $N^{\prime} R^{\prime} D$.

\section{Materials and methods}

The compound 7,8 benzo-4-azidomethyl coumarin (7BAMC) was synthesized using standard methods [21-23] and the molecular structure of the same is shown in Fig. 1. The quencher aniline was obtained from S.D. Fine Chemicals Limited and was tested for its purity before use. The HPLC grades of all solvents (S. D. Fine Chemicals Ltd) were used without further purification to prepare the solution. However, the purity of the solvents was checked by the 


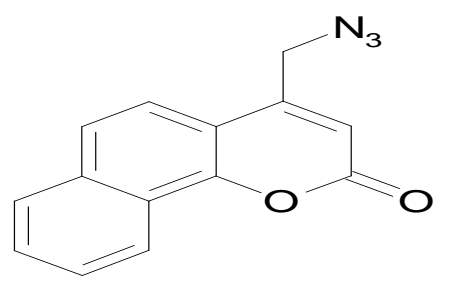

Fig. 1. Molecular structure of 7,8 benzo-4-azidomethyl coumarin (7BAMC)

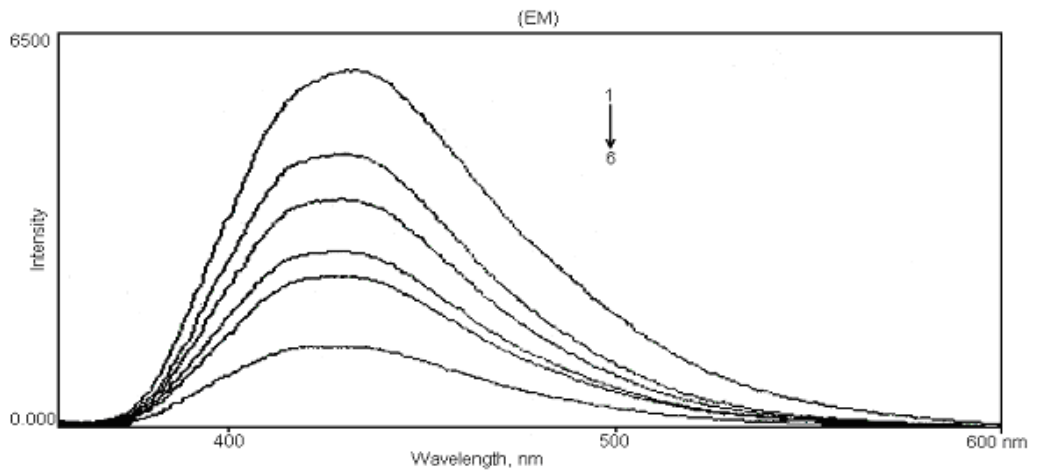

Fig.2 Fluorescence emission spectra of 7BAMC $(C=1 \times 10-4 \mathrm{M} / \mathrm{L}, \lambda \operatorname{exc}=350 \mathrm{~nm})$ in the presence of aniline in tetrahydrofuran at $27^{\circ} \mathrm{C}$. Concentrations of aniline (in M/L) (1) 0.00 (2) 0.02 (3) 0.04 (4) 0.06 (5) 0.08 (6) 0.1

background fluorescence. The solutions were prepared by keeping the concentration of $1 \times 10^{-4} \mathrm{M} / \mathrm{L}$ and varying the quencher concentration $(0.02 \mathrm{M}$ to $0.10 \mathrm{M})$ in all the solvents. Fluorescence intensities of the solutions were measured on Hitachi F2000 Spectrofluorimeter at room temperature with perpendicular geometry. Fluorescence measurements were made by taking fresh solution each time in a rectangular quartz cell having an airtight stopper. The solute has maximum absorption at $350 \mathrm{~nm}$. The solute was excited at $350 \mathrm{~nm}$ and the fluorescence spectra were recorded. The maximum fluorescence corresponds to $440 \mathrm{~nm}$. First the fluorescence intensity $I_{0}$ was measured without the quencher and then the fluorescence intensity I was measured at different quencher concentrations at a fixed solute concentration. The experimental values are reproducible within $5 \%$ of the experimental error. 


\section{Results and discussion}

The S-V plots obtained using the experimentally determined values of $I o$ and $I$ are found to be nonlinear in all the solvents showing positive deviation and are shown in Fig. 3. Similar experimental results were also observed by others [5-8]. Thus, positive deviation from linearity suggests that quenching mechanism is not purely collisional and this may be attributed either to the ground state complex formation or sphere of action static quenching models [12].

The role of ground state complex formation is ruled out in the present case. This is also confirmed by the fact that there is no shift in the peak position in the emission spectrum of 7BAMC in tetrahydrofuran with different quencher concentrations and the emission spectra of the same is shown in Fig. 2. The analysis of the data for positive deviation in S-V plots was made using "Sphere of action static quenching model". Fig. 4 shows the plots of [1-(I/IO ) ] / $\mathrm{Q}]$ against I/ $\mathrm{I}_{\mathrm{O}}$ for 7BAMC with aniline as quencher for different solvents and these plots are linear according to eqn. (6).

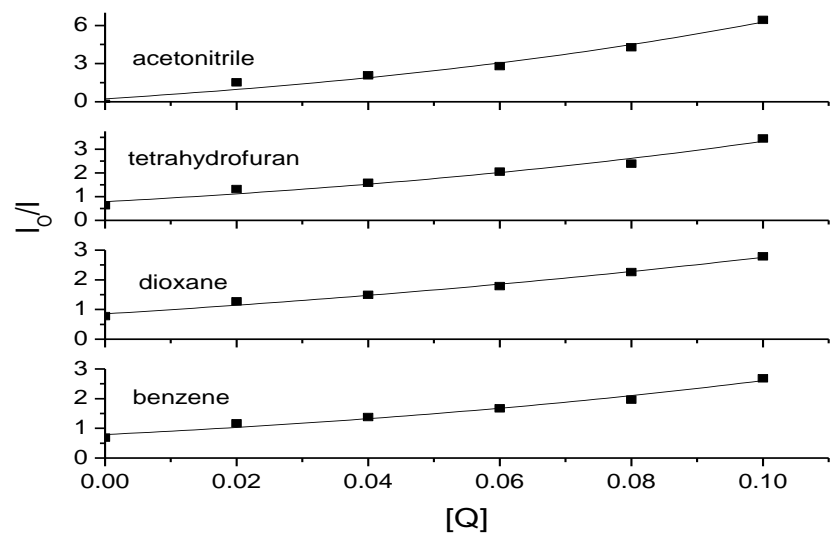

Fig. 3. Stern-Volmer plot of I0/I against [Q] in different solvents of 7BAMC + aniline system. 


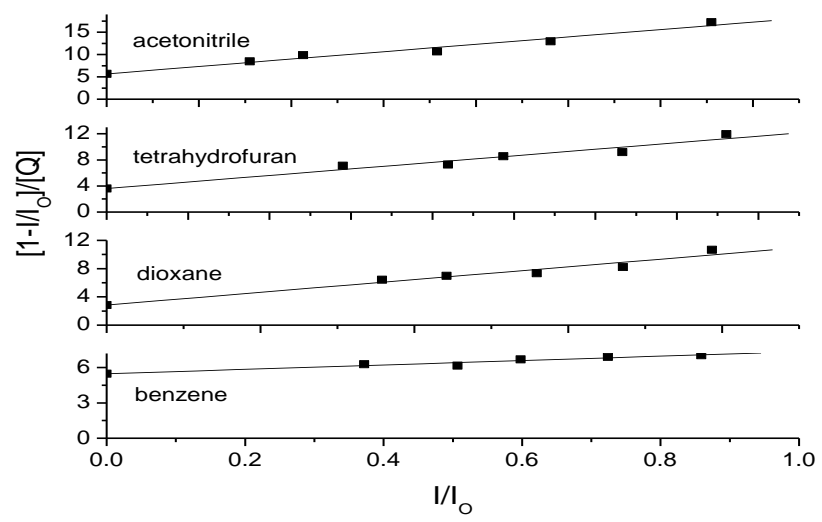

Fig. 4. Modified Stern-Volmer plot of (1-I/I0)/[Q] against I/I0 in different solvents of 7BAMC + aniline system.

The dynamic quenching constant $\mathrm{K}_{\mathrm{sv}}$ is determined in all the cases by least square fit method using equation (6) and the values are given in Table 1. Fluorescence lifetime $\tau$ of the solute was measured using Photophysics model of TCSPC nanosecond fluorescence spectrometer and data is given at the bottom of Table 1. Bimolecular quenching parameter $\mathrm{k}_{\mathrm{q}}$ was determined from the experimentally determined values of $\mathrm{K}_{\mathrm{Sv}}$ and $\tau$ according to the relation $\mathrm{k}_{\mathrm{q}=} \mathrm{K}_{\mathrm{SV} / \tau} \tau$ and these values are given in Table 1 .

In order to support static and dynamic (transient) effects, we have determined the magnitudes of static quenching constant $\mathrm{V}$ and radii $r$ of sphere of action (or kinetic distance) using the abovementioned theory for sphere of action model. With the use of eqs. (4), (5) and (6) the values of $\mathrm{V}$ and $\mathrm{r}$ are determined by least square fit method in all the systems and the values of $\mathrm{V}$ and $\mathrm{r}$ are given in Table 1. Similar results were also obtained by others [5-8].

The radii of the solute $\left(R_{Y}\right)$ and the quencher $\left(R_{Q}\right)$ molecules were determined by adding the atomic volumes of all the atoms constituting the molecule as suggested by Edward [24] and are given at the bottom of Table 1 . From these values of $R_{Y}$ and $R_{Q}$ the sum of the molecular radii (R) of the solute 7BAMC and the quencher aniline is determined. This sum of the molecular radii $\mathrm{R}$ is referred as encounter distance or contact distance or reactive distance. This value of $\mathrm{R}$ is then compared with the values of ' $r$ ' to verify whether the reaction is due to "sphere of action model" or 
not. Since according to Andre et al. [11] and Zeng et al. [16], the static effect takes place especially in the case of steady state experiments irrespective of the ground state complex formation provided the reactions are diffusion limited. From Table 1, we see that the values of kinetic distance $(r)$ are larger than the encounter distance $(\mathrm{R})$ indicating that the sphere of action model holds good in our case also. Further it may also be noted that a positive deviation in the $\mathrm{S}-\mathrm{V}$ plot is expected when both static and dynamic quenching occurs simultaneously [5-8].

\begin{tabular}{|l|r|r|r|r|r|r|}
\hline \multicolumn{1}{|c|}{ Solvent } & $\begin{array}{c}\mathrm{K}_{\mathrm{SV}} \\
\left(\mathrm{m}^{-1}\right)\end{array}$ & $\begin{array}{c}\mathrm{Kq} \mathrm{x} \mathrm{10}^{-10} \\
\left(\mathrm{~m}^{-1} \mathrm{~s}^{-1}\right)\end{array}$ & Intercept & Range of W & $\begin{array}{c}\mathrm{V}_{\left(\mathrm{mole}^{-1}\right.} \\
\left.\mathrm{dm}^{3}\right)\end{array}$ & $\mathrm{r}\left(\mathrm{A}^{0}\right)$ \\
\hline Benzene & 1.869 & 0.180 & 5.474 & $0.453-0.891$ & 8.405 & 14.936 \\
\hline Dioxane & 9.015 & 0.870 & 2.844 & $0.716-0.944$ & 3.460 & 11.109 \\
\hline Tetrahydrofuran & 10.044 & 0.969 & 3.604 & $0.640-0.928$ & 4.840 & 12.426 \\
\hline Acetonitrile & 16.650 & 1.598 & 5.675 & $0.433-0.887$ & 8.920 & 15.234 \\
\hline
\end{tabular}

Table 1: The dynamic quenching constant KSV, quenching rate parameter kq, intercept ( 1-w)/Q, range of $\mathrm{W}$, static quenching constant $\mathrm{V}$ and Kinetic distance $\mathrm{r}$ for different solvents.

$$
\mathrm{R}_{\mathrm{Y}}=3.771 \mathrm{~A}^{0} \quad \mathrm{R}_{\mathrm{Q}}=2.840 \mathrm{~A}^{0} \quad \mathrm{~T}_{0}=1.036 \mathrm{~ns}
$$

In order to find out whether the reactions are diffusion limited, we invoke the finite sink approximation model. To apply this according to eqn. (14), we need to determine the values of $\mathrm{K}^{-1} \mathrm{~Sv}$ and $[\mathrm{Q}]^{1 / 3}$, where $\mathrm{K}_{\mathrm{SV}}=\left[\left(\mathrm{I} / \mathrm{I}_{\mathrm{O}}\right)-1\right] /[\mathrm{Q}]$ and $[Q]$ the quencher concentration from $0.02 \mathrm{M}$ to $0.10 \mathrm{M}$. For efficient quenching processes (concentration dependent) the value of $\mathrm{K}_{\mathrm{sv}}$ is often observed to increase with [Q]. Hence, the values of $\mathrm{K}_{\mathrm{SV}}$ were determined at each quencher concentration in all the solvents and the values of $\mathrm{K}^{-1} \mathrm{SV}$ and $[\mathrm{Q}]^{1 / 3}$ are also determined. Fig. 5 shows the plots of $\mathrm{K}^{-1} \mathrm{~Sv}$ against $[\mathrm{Q}]^{1 / 3}$. From this figure, we see plots of $\quad \mathrm{K}^{-1} \mathrm{SV}$ against $[\mathrm{Q}]^{1 / 3}$ in different solvents are almost linear and small deviations may be due to experimental uncertainties. Hence, the linear dependence of $\mathrm{K}^{-1} \mathrm{SV}$ on the onethird power of quencher concentration within the error limits is confirmed [16]. Then from the least square fit value of $\mathrm{KO}_{\mathrm{SV}}$ (SternVolmer constant at $[Q]=0$ ) was obtained from the intercept of the plot of $\mathrm{K}^{-1} \mathrm{~Sv}$ against $[\mathrm{Q}]^{1 / 3}$ according to equation (14). Similarly, the mutual diffusion coefficient $\mathrm{D}$ was determined from the slope of equation (14) by least square fit method and the values of $\mathrm{KO}_{\mathrm{Sv}}$ and $\mathrm{D}$ are given in Table 2. Using these values of $\mathrm{KO}_{\mathrm{SV}}$ and $\mathrm{D}$, the 
distance parameter $R^{\prime}$ was determined according to equation (15) and the values of $R^{\prime}$ are given in Table 2. From the values of distance parameter $R^{\prime}$ and encounter distance $R$ activation energy controlled rate constant $\mathrm{k}_{\mathrm{a}}$ was also determined according to equation (16). This value of $k_{a}$ can only be determined for $R^{\prime}$ less than $R$ [16]. In the case dioxane experimentally determined value of the distance parameter $R^{\prime}$ is less

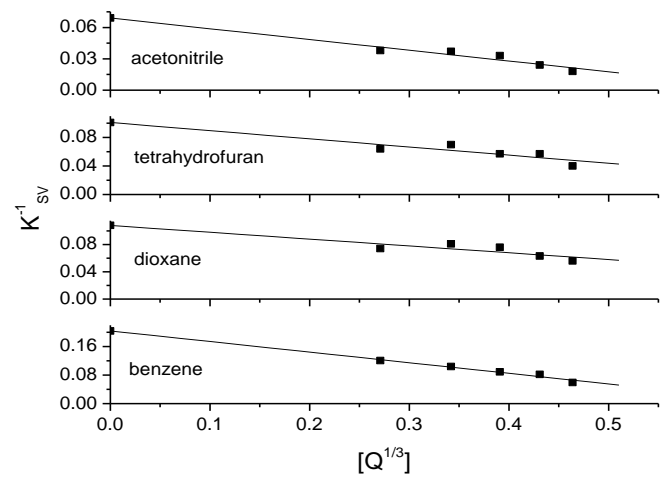

Fig. 5. Plots of K-1SV versus $[\mathrm{Q}] 1 / 3$ for $7 \mathrm{BAMC}+$ aniline system in different solvents.

than the encounter distance $R$. Hence the value of $k_{a}$ is determined only for dioxane and is given in Table 2. From Table 2, we see that $\mathrm{k}_{\mathrm{q}}$ approximately equal to $4 \pi \mathrm{N}^{\prime} \mathrm{R}^{\prime} \mathrm{D}$ and $\mathrm{k}_{\mathrm{a}}>\mathrm{k}_{\mathrm{q}}$ or alternatively $\mathrm{k}_{\mathrm{a}}>\mathrm{k}_{\mathrm{q}}$, may suggest that quenching is less efficient in dioxane and tetrahydrofuran than in the remaining solvents [11, 16]. This may be due to the fact that the activation process is more predominant in the quenching mechanism than the diffusion process. However, according to Zeng et al [16], if $k_{a}$ is greater than $k_{d}\left(=4 \pi N^{\prime} R^{\prime} D\right)$ [i.e. equation (11)] then the reactions are said to be diffusion limited. Here, the values of $k_{a}$ and $k_{d}$ are expressed in terms of D (mutual diffusion coefficient, determined by Stokes Einstein relation) because $\mathrm{D}$ is same in both cases and the $k_{d}\left(=4 \pi \mathrm{N}^{\prime} \mathrm{R}^{\prime} \mathrm{D}\right)$ value is given at the bottom of the Table 2, which is true in our case for dioxane and tetrahydrofuran. However, for remaining solvents the values of $k_{a}$ cannot be determined because the values of $R^{\prime}$ in these solvents are greater than the values of $R$. But according to Joshi et al [20], the bimolecular quenching reactions are said to be diffusion limited if the values of $\mathrm{k}_{\mathrm{q}}$ [determined from equation (6)] are greater than $4 \pi N^{\prime} R^{\prime} D$. Hence the values of $4 \pi N^{\prime} R^{\prime} D$ are calculated 
using the experimentally determined values of $R^{\prime}$ and $D$ of equation (14) and are given in Table 2. We see from Table 2 that $k_{q}$ is greater than $4 \pi N^{\prime} R^{\prime} D$ (i.e. $k_{q}>>4 \pi N^{\prime} R^{\prime} D$ ) in case of acetonitrile

\begin{tabular}{|l|l|l|l|l|l|l|}
\hline Solvent & $\begin{array}{l}\mathrm{K}_{s v}^{0} \\
\left(\mathrm{M}^{-1}\right)\end{array}$ & $\begin{array}{l}\mathrm{D} \times 10^{5} \\
\left(\mathrm{~cm}^{2}{ }^{-1}\right)\end{array}$ & $\begin{array}{l}\mathrm{R}^{\prime} \times 10^{8} \\
(\AA)\end{array}$ & $\begin{array}{l}4 \pi \mathrm{N}^{\prime} \mathrm{DR} \\
\times 10^{-10}\left(\mathrm{M}^{-1} \mathrm{~S}^{-1}\right)\end{array}$ & $\begin{array}{l}\mathrm{K}_{\mathrm{q}} \times 10^{-10} \\
\left(\mathrm{M}^{-1} \mathrm{~S}^{-1}\right)\end{array}$ & $\begin{array}{l}\mathrm{K}_{\mathrm{a}} \times 10^{-10} \\
\left(\mathrm{M}^{-1} \mathrm{~S}^{-1}\right)\end{array}$ \\
\hline \hline Benzene & 4.901 & 0.662 & 9.445 & 0.473 & 0.180 & - \\
\hline \hline Dioxane & 9.259 & 1.987 & 5.944 & 0.893 & 0.870 & 8.873 \\
\hline \hline Tetrahydrofuran & 9.900 & 1.713 & 7.373 & 0.955 & 0.969 & - \\
\hline \hline Acetonitrile & 14.492 & 1.911 & 9.674 & 1.398 & 1.598 & - \\
\hline
\end{tabular}

Table 2: The values of KOSV (Steady state quenching constant at $[\mathrm{Q}]=0$, Mutual diffusion

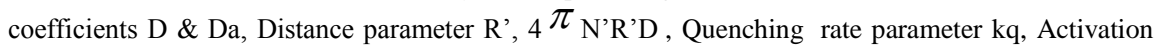
energy controlled rate constant ka, Quenching rate constant for diffusion controlled reaction kd and encounter distance $\mathrm{R}$ are given.

$$
R\left(R_{Y}+R_{Q}\right)=6.611 \AA
$$

\begin{tabular}{|c|c|c|c|}
\hline Solvent & $\mathrm{D}^{\mathrm{a}} \times 10^{5}\left(\mathrm{~cm}^{2} \mathrm{~s}^{-1}\right)$ & $\mathrm{D}^{\mathrm{b}} \times 10^{5}\left(\mathrm{~cm}^{2} \mathrm{~s}^{-1}\right)$ & $\mathrm{R}^{\prime} \times 10^{8}(\AA)$ \\
\hline Benzene & 3.520 & 0.662 & 9.445 \\
\hline Dioxane & 1.766 & 1.987 & 5.944 \\
\hline Tetrahydrofuran & 4.680 & 1.713 & 7.373 \\
\hline Acetonitrile & 5.770 & 1.911 & 9.674 \\
\hline
\end{tabular}

Table 3: The values of Mutual diffusion coefficients $\mathrm{Da}$ and $\mathrm{Db}$, distance parameter $\mathrm{R}$ ' and encounter distance $\mathrm{R}$ are given.

Da : Diffusion Coefficients determined from Stokes Einstein relation

Db: Diffusion Coefficients determined from Finite Sink Model solvent, which is an expected result for diffusion limited reaction [18].But for the remaining solvent benzene, values of $\mathrm{kq}$ are not gre ater than $4 \pi N^{\prime} R^{\prime} D$, such low value of $\mathrm{kq}$ is due to low value of diffusion coefficient (D) [20]. It appears that in the benzene solvent, efficiency of quenching is less when compared with other solvents. Hence, reduced quenching efficiency is due to decrease in diffusion coefficients of given bimolecular quenching reactions. However it may also be noted that the values of $R$ ' and ' $r$ ' kinetic distance do not change drastically. It is important to note that, when an attempt was made to correlate the experimentally determined values of mutual diffusion coefficient $\mathrm{D}$ and distance parameter $R^{\prime}$ using 
finite sink approximation model with the corresponding values of $\mathrm{D}$ and R calculated from Stoke's- Einstein and Edward's empirical relations respectively in all the systems, the values of $D$ and $R^{\prime}$ (or R) comparable only in some solvents. From Table 3 it is clear that correlation fails in some others solvents. Similar discrepancies have also been observed by others [16, 20] in some bimolecular quenching reactions. This may be due to the uncertainties in the values of adjustable parameter ' $a$ ' in the Stoke's- Einstein relation and the approximation in the values of the atomic volume in the Edward's empirical relation. Hence, we may conclude that finite sink approximation model is valid in recovering the parameters D and $\mathrm{R}^{\prime}$ (or R).

\section{Conclusion}

From the above discussion we observe that:

(1) the Stern-Volmer plots show positive deviation, indicating efficient fluorescence quenching.

(2) Static quenching constant ' $V$ ' and kinetic distance ' $r$ ' are in agreement with the corresponding literature values.

(3) The values of ka are greater than $\mathrm{kd}$ in tetrahydrofuran and acetonitrile. The values of $\mathrm{kq}$ are greater than $4 \pi \mathrm{N}^{\prime} \mathrm{R}^{\prime} \mathrm{D}$ in acetonitrile, which is an expected result for diffusion limited reaction[18]. But for remaining solvent benzene, values of $\mathrm{k}_{\mathrm{q}}$ are not greater than $4 \pi N^{\prime} R^{\prime} D$, such low value of $k_{q}$ is due to low value of diffusion coefficients (D) [20]. It appears that in the benzene solvent, efficiency of quenching is less when compared with other solvents. Hence, reduced quenching efficiency is due to decrease in diffusion coefficients of given bimolecular quenching reactions. However it may also be noted that the values of $\mathrm{R}^{\prime}$ and ' $r$ ' kinetic distance do not change drastically. (4) Finite sink approximation model is valid in recovering the parameters $D$ and $R^{\prime}(R)$. Hence, it may conclude that quenching reaction is diffusion limited and both static and dynamic (transient) quenching processes are partly playing a role in these systems. 


\section{Acknowledgements}

The author (RMM) is grateful to Dr. S. Y. Kulakarni. Principal, MSRIT, Dr. M. Suguna, HOD, Department of Physics, MSRIT and The Management of MSRIT, Bangalore for their encouragement and providing necessary requirements.

\section{References}

[1] K. K. Rothatgi-Mukherjee, Fundamental of Photochemistry, Wiley Eastern Ltd., New Delhi, India, 1986.

[2] J. R. Lackowicz, Principles of Fluorescence Spectroscopy, Plenum Press, New York, 1983.

[3] T. Moriya, "“Excited-state Reactions of Coumarins in Aqueous Solutions. II. The Fluorescence Quenching of 7-Ethoxycoumarins by Halide Ions" Bull. Chem. Soc. Jpn., vol. 57, p. 1723, 1984. http:// dx.doi.org/10.1246/bcsj.57.1723.

[4] E. A. Lissi, M. V. Encinas, S. G. Birtolotti, J. J. Cosa and C. M. Previtali, "Fluorescence Quenching of Indolic Compounds in reverse Micelles of AOT," Photochem. Photobiol, vol. 51, p. 53, 1990. http://dx.doi.org/10.1111/j.1751-1097.1990.tb01683.x.

[5] R. M. Melavanki, R. A. Kusanur, M. V. Kulakarni and J. S. Kadadevaramath, "Role of solvent polarity on the fluorescence quenching of newly synthesized 7,8-benzo-4-azidomethyl coumarin by aniline in benzene-acetonitrile mixtures", J. Lumin., vol. 128, p. 573, 2007. http://dx.doi.org/10.1016/j.jlumin.2007.08.013,.

[6] R. M. Melavanki, R. A. Kusanur, M. V. Kulakarni and J. S. Kadadevaramath, "Quenching mechanisms of 5BAMC by aniline in different solvents using Stern-Volmer plots," J. Lumin., vol. 129, p. 1298, 2009. http://dx.doi.org/10.1016/j.jlumin.2009.06.011.

[7] R. M. Melavanki, R. A. Kusanur, J. S. Kadadevaramath and M. V. Kulakarni, "Effect of Solvent Polarity on the Fluorescence Quenching of Biologically Active 5BAMC by Aniline in Binary Solvent Mixtures," J. Fluoresc., vol. 20, p. 1175, 2010. http://dx.doi.org/ 10.1007/s10895010-0664-7.

[8] N. R. Patil, R. M. Melavanki, S. B. Kapatakar, K. Chandrasekhar, H. D. Patil and S. Umapathy, "Fluorescence quenching of biologically active carboxamide by aniline and carbon tetrachloride in different solvents 
using Stern-Volmer plots," Spectrochimica Acta Part A., vol. 79, p. 1985, 2011. http://dx.doi.org/10.1016/j.saa.2011.05.104.

[9] N. R. Patil, R. M. Melavanki, S. B. Kapatakar, K. Chandrasekhar, N. H. Ayachit and S. Umapathy, "Solvent effect on the fluorescence quenching of biologically active carboxamide by aniline and carbon tetrachloride in different solvents using S-V plots", vol. 132, p. 558, 2012. http://dx.doi.org/10.1016/j.jlumin.2011.08.036.

[10]R. M. Melavanki, N. R. Patil, H. D. Patil, R. A. Kusanur and J. S. Kadadevaramath, "Steady state and time resolved methods of fluorescence quenching of three coumarin dyes using S-V plots," Ind. J. Pure and Appl. Phys., vol. 49, p. 748, 2011.

[11]J. C. Andre, M. Niclause and W. R. Ware, "Kinetics of partly diffusion controlled reactions. I. Transient and apparent transient effect in fluorescence quenching," Chem. Phys., vol. 28, p. 371, 1978. http://dx.doi.org/10.1016/0301-0104(78)80014-7.

[12]P. K. Behera and A. K. Mishra, "Static and dynamic model for 1naphthol fluorescence quenching by carbon tetrachloride in dioxaneacetonitrile mixtures," J. Photochem. Photobiol. A, vol. 71, p. 115, 1993. http://dx.doi.org/10.1016/1010-6030(93)85061-C.

[13]T. L. Nemzek and W. R. Ware, "Kinetics of diffusion-controlled reactions: Transient effects in fluorescence quenching," J. Chem, Phys., vol. 62, p. 477, 1975. http://dx.doi.org/10.1063/1.430501 .

[14]J. M. Frank and S. J. Wawilow, "Spehere of action of the extinction phenomenon in fluorescent liquids," Z. Phys, vol. 69, p. 100, 1931.

[15]H. Zeng and G. Durocher, "Analysis of fluorescence quenching in some antioxidants from non-linear Stern-Volmer plots," J. Lumin, vol. 63, pp. 75, 1995. http://dx.doi.org/10.1016/0022-2313(94)00045-E.

[16]J. Keizer, "Nonequilibrium statistical thermodynamics and the effect of diffusion on chemical reaction rates," J. Phy. Chem., vol. 86, p. 5052, 1982. http://dx.doi.org/10.1021/j100223a004.

[17]J. Keizer, "Nonlinear fluorescence quenching and the origin of positive curvature in Stern-Volmer plots," J. Am. Chem. Soc, vol. 105, p. 1494, 1983. http://dx.doi.org/10.1021/ja00344a013.

[18] J. Keiger, "Additions and Corrections - Nonlinear Fluorescence Quenching and the Origin of Positive Curvature in Stern-Volmer Plots," J. Am, Chem. Soc., vol. 107, p. 5319, 1985. http:// dx.doi.org/10.1021/ja00304a601. 
[19] J. Keizer, "Diffusion effects on rapid bimolecular chemical reactions," Chem. Rev., vol. 87, p. 167, 1987. http://dx.doi.org/ 10.1021/ cr00077a009.

[20] G. C. Joshi, R. Bhatnagar, S. Doraiswamy and N. Periasamy, "Diffusion-controlled reactions: transient effects in the fluorescence quenching of indole and N-acetyltryptophanamide in water," J. Phy. Chem., vol. 94, p. 2908, 1990. http://dx.doi.org/ 10.1021/ j100370a033.

[21] R. A. Kusanur and M. V. Kulakarni, Ind. Counc. Chem. $22^{\text {nd }}$ Conference, Roorkee (Uttaranchal), 00-29, 2003,

[22] B. B. Dey and Y. Shankaranaraayan, J. Ind. Chem. Soc, vol. 11, p. 687, 1934.

[23] S. Rangaswamy, T. R. Sheshadri and V Venkateshwaralu, Proc. Ind. Acad. Sci., vol. 13A, p. 316, 1941.

[24] J. T. Edward, "Molecular Volume. Parachor", Chem, Ind., London 774, 1956. 